\title{
Some constrained optimization problems in elementary statistics
}

\author{
Wolfgang Stadje \\ Wolfgang Stadje promovierte und habilitierte in Mathematik an der Universität Göt- \\ tingen. Er ist Professor an der Universität Osnabrück und arbeitet hauptsächlich über \\ stochastische Prozesse und ihre Anwendungen, z.B. auf Warteschlangen- und Lager- \\ haltungsmodelle, sequentielle Analysis sowie über analytische und kombinatorische \\ Probleme in der Wahrscheinlichkeitstheorie.
}

\section{Introduction}

Let $x_{1}, \ldots, x_{n} \in \mathbb{R}$ be $n$ measurements of some quantity. The following elementary functions of $x_{1}, \ldots, x_{n}$ are of particular statistical importance:

- the arithmetic mean $\bar{x}=n^{-1} \sum_{i=1}^{n} x_{i}$;

- the variance $s^{2}=n^{-1} \sum_{i=1}^{n}\left(x_{i}-\bar{x}\right)^{2}$;

- the extreme values $M=\max _{1<i<n} x_{i}$ and $m=\min _{1<i<n} x_{i}$ and the range $R=M-m$;

- in the case of positive $x_{i}$ 's, the geometric mean $\bar{x}_{g}=\left(\prod_{i=1}^{n} x_{i}\right)^{1 / n}$ and the harmonic mean $\bar{x}_{h}=n / \sum_{i=1}^{n} 1 / x_{i}$.

The arithmetic mean is certainly the most widely used (and oldest) method to combine discordant measurements in order to summarize the data in a single value. The geometric and the harmonic mean are measures of location used in special circumstances, for example to determine an average of $n$ successive price increases, or an average price if the same amount of some goods is purchased $n$ times at different prices (see for

Zur Bündelung der in $n$ Meßwerten einer Größe enthaltenen Information sind das arithmetische Mittel, die Varianz, Maximum und Minimum sowie die Spannweite die gebräuchlichsten statistischen Merkmale. Die vorliegende Arbeit geht der Frage nach, welche Werte diese statistischen Funktionen jeweils maximal annehmen können, wenn Bedingungen an die anderen gestellt werden. Mehrere solche Optimierungsaufgaben mit Nebenbedingungen haben ästhetisch ansprechende Lösungen, die mit Hilfe verschiedener elementarer Techniken hergeleitet werden. Neben dem arithmetischen werden außerdem das geometrische und das harmonische Mittel betrachtet. 
example [1]). The three means satisfy the inequalities $\bar{x} \geq \bar{x}_{g} \geq \bar{x}_{h}$. The variance is the classical measure of dispersion around the center of location, while the range gives the full amount of variability without indicating how concentrated 'most of' the measurements are. $\bar{x}$ and $s^{2}$ are also important quantities in mechanics: if a total mass 1 is distributed on the real line in portions of size $1 / n$ at the points $x_{1}, \ldots, x_{n}$, then $\bar{x}$ and $s^{2}$ are the center of gravity and the moment of inertia around this center, respectively.

It seems interesting to find the extremal values of these functions under constraints on one or several of the others, especially if the solutions turn out to have an appealing analytic form. This is indeed the case, and in this note we will solve the following six of these constrained optimization problems:

(P1) Maximize $s^{2}$ subject to $\bar{x}=\mu$ and $0 \leq x_{i} \leq c, i=1, \ldots, n$ (with given $\mu \in \mathbb{R}$ and $c>0$ ).

(P2) Maximize $s^{2}$ subject to $\bar{x}=\mu, R=r(\mu \in \mathbb{R}, r>0$ given).

(P3) Maximize $M=\max _{1 \leq i \leq n} x_{i}$ subject to $\bar{x}=\mu$ and $s^{2}=\sigma^{2}$ (with given $\mu \in \mathbb{R}$ and $\left.\sigma^{2}>0\right)$.

(P4) Maximize the range $R$ subject to $\bar{x}=\mu, s^{2}=\sigma^{2}$.

(P5) Maximize $\bar{x}_{g} / \bar{x}$ subject to $x_{1}, \ldots, x_{n}>0,1-\delta_{0} \leq x_{i} / \bar{x} \leq 1+\delta_{1}$ for given $\delta_{0} \in[0,1)$ and $\delta_{1} \in[0,1)$, not both equal to zero.

(P6) Minimize $\bar{x}_{h}$ subject to $\bar{x}=\mu, s^{2}=\sigma^{2}$ and $x_{i} \geq c, i=1, \ldots, n$ (with given $\sigma^{2}>0, c>0$ and $\left.\mu \in(c, \infty)\right)$.

Solutions. The extremal values in the above problems are given by the following formulas:

(P1) $s_{\max }^{2}=n^{-1}\left(j(c-\mu)^{2}+(n-j-1) \mu^{2}+((n-1) \mu-j c)^{2}\right)$, where the nonnegative integer $j$ is defined by $j<n \mu / c \leq j+1$.

(P2) $s_{\max }^{2}=\left\{\begin{array}{c}\frac{n^{2}-1}{4 n^{2}} r^{2} \text { if } n \text { is odd, } \\ \frac{1}{4 n} r^{2} \text { if } n \text { is even. }\end{array}\right.$

(P3) $M_{\max }=\mu+(n-1)^{\frac{1}{2}} \sigma^{2}$.

(P4) $R_{\max }=(2 n)^{\frac{1}{2}} \sigma^{2}$.

(P5) $\left(\bar{x}_{g} / \bar{x}\right)_{\max }=\left(\left(1-\delta_{0}\right)^{k}\left(1+\delta_{1}\right)^{n-k-1}\left(1+k\left(\delta_{0}+\delta_{1}\right)\right)-(n-1) \delta_{1}\right)^{-1 / n}$, where $k=\left[n \delta_{1} /\left(\delta_{0}+\delta_{1}\right)\right]$.

(P6) For this problem we can only prove that

$$
\left(\bar{x}_{h}\right)_{\min } \geq \frac{c\left(\sigma^{2}+\mu^{2}-c \mu\right)}{\sigma^{2}-\mu^{2}+c \mu} .
$$

The exact value of the minimum is unknown. 
Of course, $[a]$ is defined to be the largest integer $m$ satisfying $m \leq a$.

We will prove these results and also give the values of $x_{1}, \ldots, x_{n}$ for which the extrema are attained. Many similar problems whose solutions seem to be unknown can be easily formulated. An example is (P6); the corresponding maximization problem is also open.

A basic reference text on inequalities of the type considered here is [3]. An interesting paper in the spirit of this note is [2], where the following is proved: For any $m \geq 3$ the maximal value $\alpha_{m}$ of $n^{-1} \sum_{i=1}^{n} x_{i}^{m}$ subject to $\bar{x}=0$ and $s^{2}=1$ is attained at

$$
\left(x_{1}, \ldots, x_{n}\right)=\left((n-1)^{1 / 2},-(n-1)^{-1 / 2}, \ldots,-(n-1)^{-1 / 2}\right),
$$

bounded by $\left((n-1)^{m}+(-1)^{m}\right) / n(n-1)^{(m / 2)-1}$ and satisfies $\alpha_{2 m} \geq \alpha_{m+1}^{2}+\alpha_{m}^{2}$.

\section{Derivations}

Theorem 1 In problem (P1), choose $j$ such that $j<n \mu / c \leq j+1$. Then the maximum is attained at $x^{0}=\left(x_{1}^{0}, \ldots, x_{n}^{0}\right)$, where

$$
x_{1}^{0}=\ldots=x_{j}^{0}=c, x_{j+1}^{0}=n \mu-j c, x_{j+2}^{0}=\ldots=x_{n}^{0}=0,
$$

and is equal to

$$
s_{\max }^{2}=n^{-1}\left[j(c-\mu)^{2}+(n-j-1) \mu^{2}+((n-1) \mu-j c)^{2}\right] .
$$

Proof. Since $n s^{2}=\sum_{i=1}^{n} x_{i}^{2}-n \mu^{2}$, we have to maximize $\sum_{i=1}^{n} x_{i}^{2}$ subject to $\bar{x}=\mu$ and, without restriction of generality, $c \geq x_{1} \geq \ldots \geq x_{n} \geq 0$. Suppose that the maximum under these constraints is attained at some point $\left(u_{1}, \ldots, u_{n}\right)$ satisfying $u_{k}=c, k \leq i_{0}$, for some $i_{0} \in\{0,1, \ldots, j-1\}$ and $u_{i_{0}+1}<c$. Then

$$
u_{1}+\cdots+u_{i_{0}}+u_{i_{0}+1}<i_{0} c+c \leq j c<n \mu
$$

so that $u_{i_{0}+2}>0$. But

$$
\begin{aligned}
\left(u_{i_{0}+1}+\varepsilon\right)^{2}+\left(u_{i_{0}+2}-\varepsilon\right)^{2} & =u_{i_{0}+1}^{2}+u_{i_{0}+2}^{2}+2 \varepsilon^{2}+2 \varepsilon\left(u_{i_{0}+1}-u_{i_{0}+2}\right) \\
& >u_{i_{0}+1}^{2}+u_{i_{0}+2}^{2}
\end{aligned}
$$

for all $\varepsilon>0$. In particular, taking $\varepsilon \in\left(0, \min \left[c-u_{i_{0}+1}, u_{i_{0}+2}\right]\right)$ yields the contradiction that the maximum is not attained at $\left(u_{1}, \ldots, u_{n}\right)$.

Thus, if $x_{1}^{0} \geq \ldots \geq x_{n}^{0}$ is a maximizing point, we must have $x_{1}^{0}=\ldots=x_{j}^{0}=c$.

If $j+2 \leq n$ and $x_{j+2}^{0}>0$, reasoning as above shows that $x^{0}$ could not be a maximum. Hence, $x_{i}^{0}=0$ for $i>j+1$ and $x_{j+1}^{0}=n \mu-x_{1}^{0}-\cdots-x_{j}^{0}=n \mu-j c$. This proves (2.1), and (2.2) is now immediate. 
Theorem 2 In (P2) the maximal value is attained at

$$
x^{0}= \begin{cases}\left(\mu-\frac{r}{2}, \ldots, \mu-\frac{r}{2}, \mu+\frac{r}{2}, \ldots, \mu+\frac{r}{2}\right) & \text { if } n \text { is even }, \\ \left(\mu-\frac{n+1}{2 n} r, \ldots, \mu-\frac{n+1}{2 n} r, \mu+\frac{n-1}{2 n} r, \ldots, \mu+\frac{n-1}{2 n} r\right) & \text { if } n \text { is odd },\end{cases}
$$

and is equal to

$$
s_{\max }^{2}=\left\{\begin{array}{cl}
\frac{1}{4 n} r^{2} & \text { if } n \text { is even }, \\
\frac{n^{2}-1}{4 n^{2}} r^{2} & \text { if } n \text { is odd. }
\end{array}\right.
$$

Proof. We may assume that $\mu=0$. Let $x=\left(x_{1}, \ldots, x_{n}\right)$ be a point at which the maximum is attained. We show that up to a permutation $x$ must be of the form $(\alpha, \ldots, \alpha, \beta, \ldots, \beta)$ with $\alpha<0<\beta$. If this does not hold, then there is a nonnegative component smaller than $\max _{1 \leq i \leq n} x_{i}$ or a nonpositive component greater than $\min _{1 \leq i \leq n} x_{i}$. Thus let us suppose that one of the nonnegative $x_{i}$, say $x_{i_{0}}$, is smaller than $\max _{1 \leq i \leq n} x_{i}$.

Then all negative $x_{i}$ must be equal to $\min _{1 \leq i \leq n} x_{i}$, because if $0>x_{j_{0}}>\min _{1 \leq i \leq n} x_{i}$ for some $x_{j_{0}}$, then the point $x^{\prime}$ defined by $x_{i_{0}}^{\prime}=x_{i_{0}}+\varepsilon, x_{j_{0}}^{\prime}=x_{j_{0}}-\varepsilon, x_{i}^{\prime}=x_{i}$ for $i \neq i_{0}, j_{0}$ has the arithmetic mean $\bar{x}^{\prime}=0$ and has, for small $\varepsilon>0$, the same range as $x$, but

$$
\sum_{i=1}^{n}\left(x_{i}^{\prime}\right)^{2}=2 \varepsilon^{2}+2\left(x_{i_{0}}-x_{j_{0}}\right) \varepsilon+\sum_{i=1}^{n} x_{i}^{2}>\sum_{i=1}^{n} x_{i}^{2} \text { for all } \varepsilon>0 .
$$

Furthermore, all nonnegative $x_{i}$ except $x_{i_{0}}$ are equal to $\max _{1 \leq i \leq n} x_{i}$, because if $0 \leq x_{i_{0}} \leq$ $x_{i_{1}}<\max _{1 \leq i \leq n} x_{i}$ for some $i_{1} \neq i_{0}$, the point $x^{\prime \prime}$ defined by $x_{i_{0}}^{\prime \prime}=x_{i_{0}}-\varepsilon, x_{i_{1}}^{\prime \prime}=x_{i_{1}}+\varepsilon$ and $x_{i}^{\prime \prime}=x_{i}$ for $i \neq i_{0}, i_{1}$ has a larger sum of squares than $x$ but the same range for small $\varepsilon>0$, and mean $\bar{x}^{\prime \prime}=0$. It follows that up to a permutation $x$ is of the form

$$
(\alpha, \ldots, \alpha, \gamma, \beta, \ldots, \beta), \quad k \alpha \text { 's and } n-k-1 \beta \text { 's, }
$$

where $\alpha<0 \leq \gamma \leq \beta$ and $k \in\{1, \ldots, n-2\}$. We may assume that $\gamma=\beta$, since if $\gamma<\beta$ the point

$$
\left(\alpha-(n-1)^{-1} \varepsilon, \ldots, \alpha-(n-1)^{-1} \varepsilon, \gamma+\varepsilon, \beta-(n-1)^{-1} \varepsilon, \ldots, \beta-(n-1)^{-1} \varepsilon\right), \varepsilon>0
$$

gives the same mean value and the same range as $x$, as long as $0<\gamma+\varepsilon<\beta$, while its sum of squares is

$$
\begin{aligned}
& k \alpha^{2}+\gamma^{2}+(n-k-1) \beta^{2}+\varepsilon^{2}\left(\frac{k}{(n-1)^{2}}+1+\frac{n-k-1}{(n-1)^{2}}\right) \\
& \quad+2 \varepsilon\left(\gamma-\frac{\alpha k}{n-1}-\frac{\beta(n-k-1)}{n-1}\right)>k \alpha^{2}+\gamma^{2}+(n-k-1) \beta^{2}
\end{aligned}
$$


the inequality follows from

$$
\gamma-\frac{\alpha k}{n-1}-\frac{\beta(n-k-1)}{n-1}=\frac{n}{n-1} \gamma \geq 0
$$

(note that $k \alpha+\gamma+(n-k-1) \beta=0)$.

Thus the maximum is attained at some point $(\alpha, \ldots, \alpha, \alpha+r, \ldots, \alpha+r)$ and we have to find an $\alpha<0$ and a $k \in\{1, \ldots, n-1\}$ such that

$$
k \alpha^{2}+(n-k)(\alpha+r)^{2}
$$

is maximized subject to $k \alpha+(n-k)(\alpha+r)=0$. From this constraint we obtain

$$
\alpha=-\frac{n-k}{n} r, \quad \alpha+r=\frac{k}{n} r
$$

so that

$$
\begin{aligned}
k \alpha^{2}+(n-k)(\alpha+r)^{2} & =k\left(\frac{n-k}{n}\right)^{2} r^{2}+(n-k)\left(\frac{k}{n}\right)^{2} r^{2} \\
& =k(n-k) \frac{r^{2}}{n}
\end{aligned}
$$

If $n$ is even (odd), the maximum is attained for $k=n / 2(k=(n-1) / 2)$. A short calculation now yields the expression for $s_{\max }^{2}$. The theorem is proved.

Theorem 3 In problem (P3) the maximal value of $M$ is equal to

$$
M_{\max }=(n-1)^{1 / 2} \sigma
$$

and is attained at $x^{0}$, where

$$
x_{1}^{0}=\mu+(n-1)^{1 / 2} \sigma, x_{2}^{0}=\ldots=x_{n}^{0}=\mu-(n-1)^{-1 / 2} \sigma .
$$

Proof. By symmetry, we can equivalently maximize $x_{1}$ subject to $\bar{x}=\mu, s^{2}=\sigma^{2}$. We form the Lagrange function

$$
L\left(x, \lambda_{1}, \lambda_{2}\right)=x_{1}+\lambda_{1}(\bar{x}-\mu)+\lambda_{2}\left(s^{2}-\sigma^{2}\right) .
$$

Setting its partial derivatives equal to zero, we obtain

$$
\begin{aligned}
& 0=\frac{\partial L}{\partial x_{1}}=1+n^{-1} \lambda_{1}+2 n^{-1} \lambda_{2}\left(x_{1}-\mu\right), \\
& 0=\frac{\partial L}{\partial x_{2}}=n^{-1} \lambda_{1}+2 n^{-1} \lambda_{2}\left(x_{2}-\mu\right), \\
& 0=\frac{\partial L}{\partial x_{n}}=\quad n^{-1} \lambda_{1}+2 n^{-1} \lambda_{2}\left(x_{n}-\mu\right) .
\end{aligned}
$$


Summing these equations yields

$$
0=1+\lambda_{1}+2 n^{-1} \lambda_{2}\left(\sum_{i=1}^{n} x_{i}-n \mu\right)=1+\lambda_{1},
$$

i.e., $\lambda_{1}=-1$, so that

$$
x_{1}=\mu+\frac{1-n}{2 \lambda_{2}}, x_{2}=\ldots=x_{n}=\mu+\frac{1}{2 \lambda_{2}} .
$$

By the constraint on the variance,

$$
n \sigma^{2}=\sum_{i=1}^{n}\left(x_{i}-\mu\right)^{2}=\frac{(1-n)^{2}}{4 \lambda_{2}^{2}}+\frac{n-1}{4 \lambda_{2}^{2}} .
$$

Therefore,

$$
\lambda_{2}= \pm(2 \sigma)^{-1}(n-1)^{1 / 2} .
$$

We obtain the two points $x^{0}$ and $x^{1}$, where $x^{1}$ is defined by

$$
x_{1}^{1}=\mu-(n-1)^{1 / 2} \sigma, x_{2}^{1}=\ldots=x_{n}^{1}=\mu+(n-1)^{-1 / 2} \sigma .
$$

The Jacobian matrix of the mapping $\left(\bar{x}-\mu, s^{2}-\sigma^{2}\right)$ from $\mathbb{R}^{n}$ to $\mathbb{R}^{2}$ has rank 2 at $x^{0}$ and at $x^{1}$; hence $M_{\max }=x_{1}^{0}=\mu+(n-1)^{1 / 2} \sigma$. Note that the minimum possible value of $x_{1}$ is $\mu-(n-1)^{1 / 2} \sigma$.

Theorem 4 In problem $(\mathrm{P} 4)$ the maximum range is equal to

$$
R_{\max }=(2 n)^{1 / 2} \sigma,
$$

and is attained at the point $x^{0}$ given by

$$
x_{1}^{0}=\mu+(n / 2)^{1 / 2} \sigma, x_{2}^{0}=\mu-(n / 2)^{1 / 2} \sigma, x_{3}^{0}=\ldots=x_{n}^{0}=\mu .
$$

Proof. Change the variables to $u_{i}=x_{i}-\mu, i=1, \ldots, n$. Then we have to maximize $u_{1}-u_{2}$ subject to

$$
\bar{u}=0, \sum_{i=1}^{n} u_{i}^{2}=n \sigma^{2} .
$$

As in the proof of Theorem 2, we consider the corresponding Lagrange function

$$
L\left(u, \lambda_{1}, \lambda_{2}\right)=u_{1}-u_{2}+\lambda_{1} \sum_{i=1}^{n} u_{i}+\lambda_{2}\left(\sum_{i=1}^{n} u_{i}^{2}-n \sigma^{2}\right) .
$$


Set its derivatives equal to zero:

$$
\begin{aligned}
& 0=\frac{\partial L}{\partial u_{1}}=1+\lambda_{1}+2 \lambda_{2} u_{1}, \\
& 0=\frac{\partial L}{\partial u_{2}}=-1+\lambda_{1}+2 \lambda_{2} u_{2}, \\
& 0=\frac{\partial L}{\partial u_{3}}=\quad \lambda_{1}+2 \lambda_{2} u_{3}, \\
& 0=\frac{\partial L}{\partial u_{n}}=\quad \lambda_{1}+2 \lambda_{2} u_{n} .
\end{aligned}
$$

Adding these equations, we find that $\lambda_{1}=0$,

and

$$
u_{1}=-\left(2 \lambda_{2}\right)^{-1}, u_{2}=\left(2 \lambda_{2}\right)^{-1}, u_{3}=\ldots=u_{n}=0
$$

$$
n \sigma^{2}=\left(2 \lambda_{2}\right)^{-1}+\left(2 \lambda_{2}\right)^{-2} \text {, i.e., } \lambda_{2}= \pm(2 n)^{-1 / 2} \sigma^{-1} .
$$

It follows that the maximum of $u_{1}-u_{2}$ is attained at

(and the minimum at $-u^{0}$ ).

$$
u^{0}=\left((n / 2)^{1 / 2} \sigma,-(n / 2)^{1 / 2} \sigma, 0, \ldots, 0\right)
$$

For our next result we need the following

Lemma Let $-n \delta_{0} \leq a \leq n \delta_{1}$. The minimum of the function

$$
F\left(y_{1}, \ldots, y_{n}\right)=\prod_{i=1}^{n}\left(1+y_{i}\right),
$$

subject to the restrictions $y_{1}, \ldots, y_{n} \in\left[-\delta_{0}, \delta_{1}\right], \sum_{i=1}^{n} y_{i}=a$, is only attained at points that have at least $n-1$ components in $\left\{-\delta_{0}, \delta_{1}\right\}$.

Proof. If $n=2$, we have to minimize $F\left(y_{1}, y_{2}\right)=a y_{1}-y_{1}^{2}$ over the interval $\max \left[-\delta_{0}, a-\right.$ $\left.\delta_{1}\right] \leq y_{1} \leq \min \left[\delta_{1}, a+\delta_{0}\right]$. The minimum is attained at $y_{1}=\delta_{1}, y_{2}=a-\delta_{1}$ if $\delta_{1} \leq a+\delta_{0}$ and at $y_{1}=a+\delta_{0}, y_{2}=-\delta_{0}$ if $\delta_{1}>a+\delta_{0}$. The only other way to attain the minimum is to permute the components.

Now we proceed by induction on $n$. Suppose the assertion is true for $n-1$ for some $n \geq 3$. The function $F\left(y_{1}, \ldots, y_{n}\right)$ has no minimum on the set $A=\left\{y \in \mathbb{R}^{n} \mid \sum_{i} y_{i}=\right.$ $\left.a, \min _{i} y_{i}>-1\right\}$. Indeed, if $y \in A$, the point $y^{\prime} \in \mathbb{R}^{n}$ with components $y_{1}^{\prime}=y_{1}-\eta$, $y_{2}^{\prime}=y_{2}+\eta$ and $y_{i}^{\prime}=y_{i}$ for $i>2$ is in $A$ for small $|\eta|$, and $F\left(y^{\prime}\right)=F(y)+\left[\eta\left(y_{1}-\right.\right.$ $\left.\left.y_{2}\right)-\eta^{2}\right] \prod_{i=3}^{n}\left(1+y_{i}\right)$. By suitable choice of $\eta$ (positive or negative and close enough to zero) we obtain $F\left(y^{\prime}\right)<F(y)$. It follows that any absolute minimum $\left(u_{1}, \ldots, u_{n}\right)$ of $F\left(y_{1}, \ldots, y_{n}\right)$ subject to $y_{1}, \ldots, y_{n} \in\left[-\delta_{0}, \delta_{1}\right], \sum_{i} y_{i}=a$ must lie on the boundary, i.e., must have one component in $\left\{-\delta_{0}, \delta_{1}\right\}$. Assume that $u_{n}=\delta_{1}$. Then $\left(u_{1}, \ldots, u_{n-1}\right)$ is an absolute minimum of $\prod_{i=1}^{n-1}\left(1+y_{i}\right)$, subject to $y_{i} \in\left[-\delta_{0}, \delta_{1}\right], \sum_{i=1}^{n-1} y_{i}=a-\delta_{1}$. Since there are $u_{1}, \ldots, u_{n-1} \in\left[-\delta_{0}, \delta_{1}\right]$ satisfying $\sum_{i=1}^{n-1}=a-\delta_{1}$, it is clear that $-(n-1) \delta_{0} \leq a-\delta_{1} \leq(n-1) \delta_{0}$. Hence we can apply the induction hypothesis and conclude that among $u_{1}, \ldots, u_{n-1}$ at least $n-2$ are in $\left\{-\delta_{0}, \delta_{1}\right\}$. 
Theorem 5 In problem (P5) the maximal value of $\bar{x}_{g} / \bar{x}$ under the given restrictions is equal to

$$
\left(\left(1-\delta_{0}\right)^{k}\left(1+\delta_{1}\right)^{n-k-1}\left(1+k\left(\delta_{0}+\delta_{1}\right)-(n-1) \delta_{1}\right)\right)^{-1 / n},
$$

where $k=\left[n \delta_{1} /\left(\delta_{0}+\delta_{1}\right)\right]$, and is attained at

$$
x_{i}-\bar{x}= \begin{cases}-\bar{x} \delta_{0} & i=1, \ldots, k \\ \bar{x} \delta_{1} & i=k+1, \ldots, n-1, \\ \bar{x}\left(\left(\delta_{0}+\delta_{1}\right) k-(n-1) \delta_{1}\right) & i=n .\end{cases}
$$

Proof. Let $y_{i}=x_{i}-\bar{x}, i=1, \ldots, n$. We may assume that $\bar{x}=1$ and then have to minimize

$$
F\left(y_{1}, \ldots, y_{n}\right)=\prod_{i=1}^{n}\left(1+y_{i}\right)
$$

subject to

$$
y_{1}, \ldots, y_{n} \in\left[-\delta_{0}, \delta_{1}\right], \sum_{i=1}^{n} y_{i}=0
$$

Let the minimum be attained at $\left(u_{1}, \ldots, u_{n}\right)$. By the lemma, at least $n-1$ of the $u_{i}$ 's are in $\left\{-\delta_{0}, \delta_{1}\right\}$. Suppose (without loss in generality) that $-\delta_{0}$ occurs $k$ times, $\delta_{1}$ occurs $\ell$ times and that the $-\delta_{0}$ 's are left of the $\delta_{1}$ 's: $u_{1}=u_{2}=\ldots=u_{k}=-\delta_{0}$ and $u_{k+1}=\ldots=u_{k+\ell}=\delta_{1}$, where $k+\ell \in\{n-1, n\}$.

Case 1. Let $k+\ell=n-1$. Then it follows that

$$
k \delta_{0}-\ell \delta_{1}=-\sum_{i=1}^{n-1} y_{i}=y_{n} \in\left[-\delta_{0}, \delta_{1}\right] \text {. }
$$

But (2.5) implies that

$$
k \leq n \delta_{1} /\left(\delta_{0}+\delta_{1}\right) \leq k+1 .
$$

If $n \delta_{1} /\left(\delta_{0}+\delta_{1}\right)$ is not an integer, then, by (2.6), $k=\left[n \delta_{1} /\left(\delta_{0}+\delta_{1}\right)\right]$. If $n \delta_{1} /\left(\delta_{0}+\delta_{1}\right)$ is an integer, then $n \delta_{1} /\left(\delta_{0}+\delta_{1}\right) \in\{k, k+1\}$. Assume that $n \delta_{1} /\left(\delta_{0}+\delta_{1}\right)=k+1$. Then $\ell=n-1-k=n \delta_{0} /\left(\delta_{0}+\delta_{1}\right)$ and, by $(2.5)$,

$$
u_{n}=k \delta_{0}-\ell \delta_{1}=\left(\frac{n \delta_{1}}{\delta_{0}+\delta_{1}}-1\right) \delta_{0}-\frac{n \delta_{0}}{\delta_{0}+\delta_{1}} \delta_{1}=-\delta_{0},
$$

which contradicts the definition of $k$. Thus $n \delta_{1} /\left(\delta_{0}+\delta_{1}\right)=k$.

Case 2. Let $k+\ell=n$. Then

$$
0=k \delta_{0}-\ell \delta_{1}=k \delta_{0}-(n-k) \delta_{1},
$$

so that $k=n \delta_{1} /\left(\delta_{0}+\delta_{1}\right)$.

Hence $k=\left[n \delta_{1} /\left(\delta_{0}+\delta_{1}\right)\right]$ in both cases, and there are at least $n-k-1$ components equal to $\delta_{1}$. The $n$-th component is given by $\sum_{i} u_{i}=0$, i.e.,

$$
u_{n}=\left[n \delta_{1} /\left(\delta_{0}+\delta_{1}\right)\right]\left(\delta_{0}+\delta_{1}\right)-(n-1) \delta_{1} .
$$

The rest of the proof is now straightforward. 
Theorem 6 The minimum of $\bar{x}_{h}$ in problem (P6) satisfies

$$
\left(\bar{x}_{h}\right)_{\min } \geq \frac{c\left(\sigma^{2}+\mu^{2}-c \mu\right)}{\sigma^{2}-\mu^{2}+c \mu} .
$$

Proof. The problem is equivalent to finding an upper bound for $n^{-1} \sum_{i=1}^{n} 1 / x_{i}$ under the given constraints. Let $H(x)=1 / x, x>0$. Suppose that some parabola $P(x)=$ $a_{0} x^{2}+a_{1} x+a_{2}$ satisfies

$$
H(x) \leq P(x) \text { for all } x \in[c, \infty)
$$

Then

$$
n^{-1} \sum_{i=1}^{n} \frac{1}{x_{i}}=n^{-1} \sum_{i=1}^{n} H\left(x_{i}\right) \leq n^{-1} \sum_{i=1}^{n} P\left(x_{i}\right)
$$

yielding an upper bound containing $\mu, \sigma^{2}$ and the coefficients of $P$. For any $x_{0} \in[c, \infty)$ and any $a>0$, the special parabola

$$
P_{x_{0}, a}(x)=a\left(x-x_{0}\right)^{2}-x_{0}^{-2}\left(x-x_{0}\right)+x_{0}^{-1}
$$

is tangential to the hyperbola $H$ at $x_{0}$, i.e., has a double point of intersection with $H$ at $x_{0}$. It is easy to check that $H$ and $P_{x_{0}, a}$ only intersect at $x_{0}$ and at $b=\left(a x_{0}^{2}\right)^{-1}$. Therefore, if $b \leq c$, inequality (2.8) holds. Let $a=\left(c x_{0}^{2}\right)^{-1}$. Then $b=c$, so that we can use $P_{x_{0}, a}$ in (2.9) and obtain

$$
\begin{aligned}
n^{-1} \sum_{i=1}^{n} \frac{1}{x_{i}} & \leq n^{-1} \sum_{i=1}^{n}\left[\left(c x_{0}^{2}\right)^{-1}\left(x_{i}-x_{0}\right)^{2}-x_{0}^{-2}\left(x_{i}-x_{0}\right)+x_{0}^{-1}\right] \\
& =\left(c x_{0}^{2}\right)^{-1}\left(n^{-1} \sum_{i=1}^{n} x_{i}^{2}+x_{0}^{2}-2 x_{0} \mu\right)-x_{0}^{-2} \mu+2 x_{0}^{-1} \\
& =\left(c x_{0}^{2}\right)^{-1}\left(\sigma^{2}+\mu^{2}+x_{0}^{2}-2 x_{0} \mu\right)-x_{0}^{-2} \mu+2 x_{0}^{-1} \\
& =\frac{\sigma^{2}+\mu^{2}-c \mu}{c x_{0}^{2}}-\frac{2}{x_{0}}\left(\frac{\mu}{c}-1\right)+\frac{1}{c}
\end{aligned}
$$

for all $x_{0} \geq c$. As a function of $x_{0} \in[c, \infty)$, the right-hand side of (2.9) is minimal for

$$
x_{0}=\left(\sigma^{2}+\mu^{2}-c \mu\right) /(\mu-c)
$$

note that the value of $x_{0}$ given in (2.11) is well-defined and in $(c, \infty)$, because $\mu>c$ and $\sigma^{2}+\mu^{2}-c \mu>(\mu-c)^{2}+c \mu-c^{2} \geq c(\mu-c)$. Inserting this $x_{0}$ in (2.10), the right-hand side becomes

$$
\frac{1}{c}\left(1-\frac{(\mu-c)^{2}}{\sigma^{2}+\mu^{2}-c \mu}\right),
$$

which yields the lower bound in (2.7). The theorem is proved. 


\section{References}

[1] Bosch, K.: Statistik-Taschenbuch. Oldenbourg, München und Wien 1992.

[2] Lakshmanamurti, M.: On the upper bound of $\sum_{i=1}^{n} x_{i}^{m}$ subject to the conditions $\sum_{i=1}^{n} x_{i}=0$ and $\sum_{i=1}^{n} x_{i}^{2}=n$. Math. Student 18 (1950), 111-116.

[3] Mitrinović, D.S.: Analytic Inequalities. Springer, Berlin etc. 1970

Wolfgang Stadje

Universität Osnabrück

Fachbereich Mathematik/Informatik

D-49069 Osnabrück, Deutschland

e-mail: wolfgang@mathematik.uni-osnabrueck.de 